Onkologie 1996;19:380-382

\title{
Contents, Vol. 19, No. 5, 1996
}

\section{Inhalt}

ONKOLOGIE

Vol. 19, Issue 5, October 1996

Vol. 19, Heft 5, Oktober 1996

Review Articles

Übersichtsarbeiten

385 Breast Cancer and the Eye. A Literature Review

Debois, J. M.; Haustrate, F. M. (Antwerp, Belgium)

394 Advances for Sphincter Preservation in the Surgical Therapy of Rectal Cancer

Schlag, P. M. (Berlin)

399 Conservation Therapy in Patients with Head and Neck Carcinoma

Stenson, K. M; Vokes, E. E. (Chicago, IL)

404 New Clinical Trial Designs for Phase I Studies in

Hematology and Oncology: Principles and Practice of the Continual Reassessment Model

Hanauske, A.-R. (München); Edler, L. (Heidelberg)

385 Das Mammakarzinom und das Auge. Literaturübersicht

Debois, J. M;; Haustrate, F. M. (Antwerpen, Belgien)

394 Verbesserte Möglichkeiten der Sphinktererhaltung in der operativen Therapie des

Rektumkarzinoms

Schlag, P.M. (Berlin)

399 Organerhaltung bei Patienten mit Kopf-Hals-Karzinomen

Stenson, K. M.; Vokes, E. E. (Chicago, IL)

404 Neue Konzepte für Phase-I-Studien in Hämatologie und Onkologie: Prinzipien und Anwendung des «Continual Reassessment Model»

Hanauske, A.-R. (München); Edler, L. (Heidelberg)

Original Articles

Originalarbeiten

410 Weekly High-Dose 5-Fluorouracil 24-Hour Infusion and Intermediate-Dose Folinic Acid Bolus in Metastatic Colorectal Cancer

Stoffregen, C; Zurborn, K.-H.; Boehme, V; Schmid, A.; Lorenz, G.; Arendt, T.; Fölsch, U. R. (Kiel)

416 Phase II Study of Weekly High-Dose 5-Fluorouracil and Folinic Acid plus Biweekly Alternating Cisplatin and Epirubicin (FUFACE) in Patients with Advanced Gastric Carcinoma Stahl, M.; Vanhoefer, U. (Essen); Fink, U. (München); Korn, M.; Eigler, F. W. (Essen); Siewert, J. R. (München); Seeber, S.; Wilke, H. (Essen) 
419 Pattern of Failure in Long-Term Survivors after

Radio-Chemotherapy for Inoperable Head and Neck Cancer

Wendt, T.G.; Panzer, M.; Wustrow, T.P. U; Hartenstein, R. (München)

424 Perioperative Changes in Body Composition and Metabolism in Patients with Colorectal Cancer according to Tumor Stage

Weimann, A.; Raab, R.; Selberg, O.; Bischoff, S.; Bornemann, K. (Hannover); Müller, M. J. (Kiel); Meyer, H.-J. (Hannover)

430 Effect of Tamoxifen and Estrogen Treatment on the Radiosensitivity of MCF-7 Breast

Cancer Cells

Böhning, K. (Göttingen); Busch, M. (München); Rave-Frank, M. (Göttingen); Dühmke, E. (München)

410 Wöchentliche Hochdosis-5-Fluorouracil-24-Stunden-Dauerinfusion kombiniert mit intermediär dosiertem Folinsäurebolus bei metastasiertem Dickdarmkarzinom

Stoffregen, C; Zurborn. K.-H.; Boehme. V.; Schmid. A.; Lorenz, G; Arendt, T.; Fölsch, U. R.

(Kiel)

416 Phase-II-Studie mit wöchentlich hochdosiertem

5-Fluorouracil und Folinsäure sowie zweiwöchentlich alternierend Cisplatin und Epirubicin

(FUFACE) bei Patienten mit fortgeschrittenem Magenkarzinom

Stahl. M.; Vanhoefer, U. (Essen); Fink, U. (München); Korn, M.; Eigler, F W. (Essen); Siewert.

J. R. (München); Seeber, S.; Wilke, H. (Essen)

419 Rezidivanalyse nach simultaner

Radio-Chemotherapie inoperabler Kopf-Hals-Karzinome

Wendt, T. G; Panzer, M.; Wustrow, T. P. U; Hartenstein, R. (München)

424 Perioperative Veränderungen von Körperzusammen-setzung und Stoffwechsel bei Patienten mit kolorektalem Karzinom in Abhängigkeit vom Tumorstadium

Weimann, A.; Raab, R.; Selberg, O.; Bischoff, S.; Bornemann, K. (Hannover); Müller, M. J.

(Kiel); Meyer, H.-J. (Hannover)

430 Wirkung von Tamoxifen und Östrogen auf die Strahlen-empfindlichkeit von MCF-7-

Mammakarzinomzellen

Böhning, K. (Göttingen); Busch, M. (München); Rave-Fränk, M. (Göttingen); Dühmke, E.

(München)

Casuistic Contributions

Kasuistiken

437 Treatment of Prolymphocytic Leukemia with Pentostatin (Nipent@): A Case Report

Wilier, A.; Käfer, G; Queißer, W.; Hehlmann, R.; Hastka, J. (Mannheim)

440 Hodgkin's Disease after Splenic Immunocytoma: Rare Manifestation of a Dual Lymphoma

Ranft, K.; Reuter, H. (Penzberg)

437 Behandlung der Prolymphozyten-Leukämie mit Pentostatin (Nipent ${ }^{\circledR)}$ ): Eine Kasuistik

Wilier, A.; Käfer, G; Queißer. W.; Hehlmann. R.; Hastka, J. (Mannheim)

440 Morbus Hodgkin nach milzdominantem Immunozytom Seltene Form eines

Doppellymphoms

Ranft, K.; Reuter, H. (Penzberg)

Continued on page 382 
Fortsetz,ung aufSeite 382

Bibliographischer Hiiiweis: Inhaltsverzeichnisse dieser Zeitschrift erscheinen regelmäßig in current contents ${ }^{\circledR}$ sowie in anderen bibliographischen Diensten.

Contents · Inhalt

OHKOLOGIE

Vol. 19, Issue 5, October 1996

Vol. 19, Heft 5, Oktober 1996

444 Facial Angioedema Associated with Granulocyte Colony-Stimulating Factor (G-CSF)

Treatment

Laurent, D.; Schmidberger, H.; Pradier, O.; Hess, C. F. (Göttingen)

444 Faziales Angioödem nach Behandlung mit

Granulozyten-Kolonie-stimulierendem Faktor (G-CSF)

Laurent, D.; Schmidberger, H.; Pradier, O.; Hess, C. F. (Göttingen)

Clinical Information

Klinische Information

446 Resolution concerning the Development of Medical Oncology and Hematology in Germany Kleeberg, U. R., et al. (Hamburg)

446 Resolution zur Entwicklung der internistischen Onkologie und Hämatologie in Deutschland Kleeberg, U. R., et al. (Hamburg)

450 Book Reviews

452 Industrial Forum

455 Reports of Oncological Societies 379 Imprint

Instructions to Authors (inside back cover)

450 Buchbesprechungen

452 Industrieforum

455 Mitteilungen onkologischer Gesellschaften

379 Impressum

Hinweise für Autoren (3. Umschiagseite)

Contents of Next Issues

Review Articles Methods to Detect MDR1/P-Glycoprotein in Clinical Tumor

Samples

Lehnert, M. (St. Gallen)

Ibandronate: Preclinical Overview, and Malignant Hypercakemia in Humans

Bauss, E; Herrmann, Z. (Mannheim)

The Cell Cycle - Theory and Application to Cancer

Parwaresch, R.; Rudolph, P. (Kiel

Consensus

Leitlinien zur Therapie des Ösophaguskarzinoms

Junginger, T. (Mainz)

Leitlinien zur Therapie des exokrinen Pankreaskarzinoms

Junginger, T. (Mainz) 